\title{
Time evolution of electric fields and currents and the generalized Ohm's law
}

\author{
V. M. Vasyliūnas \\ Max-Planck-Institut für Sonnensystemforschung, 37191 Katlenburg-Lindau, Germany
}

Received: 1 January 2005 - Revised: 24 February 2005 - Accepted: 3 March 2005 - Published: 3 June 2005

\begin{abstract}
Fundamentally, the time derivative of the electric field is given by the displacement-current term in Maxwell's generalization of Ampère's law, and the time derivative of the electric current density is given by the generalized Ohm's law. The latter is derived by summing the accelerations of all the plasma particles and can be written exactly, with no approximations, in a (relatively simple) primitive form containing no other time derivatives. When one is dealing with time scales long compared to the inverse of the electron plasma frequency and spatial scales large compared to the electron inertial length, however, the time derivative of the current density becomes negligible in comparison to the other terms in the generalized Ohm's law, which then becomes the equation that determines the electric field itself. Thus, on all scales larger than those of electron plasma oscillations, neither the time evolution of $\boldsymbol{J}$ nor that of $\boldsymbol{E}$ can be calculated directly. Instead, $\boldsymbol{J}$ is determined by $\boldsymbol{B}$ through Ampère's law and $\boldsymbol{E}$ by plasma dynamics through the generalized Ohm's law. The displacement current may still be non-negligible if the Alfvén speed is comparable to or larger than the speed of light, but it no longer determines the time evolution of $\boldsymbol{E}$, acting instead to modify $\boldsymbol{J}$. For theories of substorms, this implies that, on time scales appropriate to substorm expansion, there is no equation from which the time evolution of the current could be calculated, independently of $\nabla \times \boldsymbol{B}$. Statements about change (disruption, diversion, wedge formation, etc.) of the electric current are merely descriptions of change in the magnetic field and are not explanations.
\end{abstract}

Keywords. Magnetospheric physics (Magnetospheric configuration and dynamics; Storms and substorms) - Space plasma physics (Kinetic and MHD theory)

\section{Introduction}

Planetary magnetospheres provide plenty of examples to illustrate Eddington's dictum: "The realities of physics are

Correspondence to: V. M. Vasyliūnas

(vasyliunas@mps.mpg.de) inhomogeneities, happenings, changes" (Eddington, 1933). Prominent in any list of outstanding research topics are such intrinsically time-dependent phenomena as magnetospheric substorms (particularly their onset and expansion phases), magnetic storms at Earth, changes and fluctuations of the aurora, or the periodic and aperiodic variations of the magnetosphere at Jupiter. Much of what we know about these phenomena is derived from observations of the magnetic field (traditionally interpreted in terms of changing electric currents) as well as of charged particles (generally assumed to be accelerated by changing electric fields). How to calculate the time derivatives of the electric field $\boldsymbol{E}$ and the electric current density $\boldsymbol{J}$ would seem therefore to be a topic of overriding importance, yet it is often dealt with in a rather qualitative ad hoc manner. The purpose of this paper is to examine what can be said about the time evolution of $\boldsymbol{E}$ and $\boldsymbol{J}$, starting from the fundamental equations and taking into account the constraints imposed by the presence of plasma.

\section{Fundamental evolutionary equations}

It is a remarkable property of classical (non-quantum) physics that all of its governing equations, with the exception of the three divergence equations of the electromagnetic and gravitational fields, can be written in the evolutionary form

$\partial Q_{k} / \partial t=F_{k}\left(Q_{1}, Q_{2}, Q_{3}, \ldots\right)$

where the $Q$ 's are all the quantities describing the system and the $F$ 's are functions of the $Q$ 's and their spatial derivatives at a given time; thus all the time derivatives are determined, solely and completely, by values at the present time (e.g. Vasyliūnas, 2001).

\subsection{Magnetic field}

The simplest of the evolutionary equations is that for the magnetic field, given by one of Maxwell's equations:

$\partial \boldsymbol{B} / \partial t=-c \nabla \times \boldsymbol{E}$ 
(Gaussian units are used throughout this paper). The rate of change of the magnetic field is thus determined by the curl of the electric field. (At least within the magnetosphericionospheric community, this is not a common way of putting it!)

\subsection{Electric field}

The displacement current in Maxwell's equations provides the evolutionary equation for the electric field:

$\partial \boldsymbol{E} / \partial t=-4 \pi \boldsymbol{J}+c \nabla \times \boldsymbol{B}$.

The rate of change of the electric field is determined by the difference between the electric current $\boldsymbol{J}$ and the curl of the magnetic field - two quantities usually treated as equal! (The contrast between the straightforward direct meaning of the two equations, Eqs. (2) and (3), and what is often taken as the "common-sense" view illustrates why a careful discussion of time evolution is needed.)

\subsection{Electric current density}

The current density $\boldsymbol{J}$ is given by

$\boldsymbol{J}=\sum_{a} q_{a} \int d^{3} v \mathbf{v} f_{a}(\mathbf{v})$

where $f_{a}(\mathbf{v})$ is the velocity distribution function of charged particles of species $a$. The equation for the time evolution of $\boldsymbol{J}$, determined by summing the motions of all the charged particles, can be calculated from the appropriate sum of velocity-moment equations (see, e.g. Rossi and Olbert, 1970; Greene, 1973):

$$
\begin{gathered}
\partial \boldsymbol{J} / \partial t=\sum_{a}\left\{\left(q_{a}^{2} n_{a} / m_{a}\right)\left(\boldsymbol{E}+\boldsymbol{V}_{a} \times \boldsymbol{B} / c\right)\right. \\
\left.-\left(q_{a} / m_{a}\right) \nabla \cdot \boldsymbol{\kappa}_{a}+q_{a} n_{a} \mathbf{g}\right\}+(\delta \boldsymbol{J} / \delta t)_{\text {coll }}
\end{gathered}
$$

where $q_{a}, m_{a}, n_{a}, \boldsymbol{V}_{a}$, and $\boldsymbol{\kappa}_{a}$ are the charge, mass, concentration, bulk velocity, and kinetic tensor, respectively, of species $a$, and $(\delta \boldsymbol{J} / \delta t)_{\text {coll }}$ represents the sum of all collision effects. The gravitational acceleration $\mathbf{g}$ has been included for exactness but mostly is unimportant in practice. The collision term can, for the purposes of this paper, be just represented symbolically (for explicit expressions in the ionosphere, see, e.g. Song et al., 2001, 2005; Vasyliūnas and Song, 2005).

Equation (5) is the exact form of what is better known, under various reformulations and approximations, as the generalized Ohm's law. The different, mostly equivalent forms as well as the multiplicity of terms sometimes encountered come from decomposing the divergences of the kinetic tensors that appear in the equation into flow and pressure components, or from substituting terms out of the plasma momentum equation (thereby introducing additional time derivatives). In its primitive form Eq. (5), the generalized Ohm's law contains no time derivatives other than $\partial \boldsymbol{J} / \partial t$ on the left-hand side. Note that this is a partial time derivative; the convective (inertial) terms $\nabla \cdot(\boldsymbol{J} \boldsymbol{V}+\boldsymbol{V} \boldsymbol{J})$ often discussed are part of the kinetic-tensor terms on the right-hand side.

\section{The large-scale (plasma) limit}

Maxwell's equations can also be used to obtain another equation for the time derivative of the current density, in terms only of the electric field:

$$
\partial \boldsymbol{J} / \partial t=-\left(c^{2} / 4 \pi\right)\left\{\nabla \times(\nabla \times \boldsymbol{E})+\left(1 / c^{2}\right) \partial^{2} \boldsymbol{E} / \partial t^{2}\right\}
$$

This is not an evolutionary equation for $\boldsymbol{J}$ because it contains higher-order derivatives on the right-hand side; in purely electromagnetic problems where $\boldsymbol{J}$ is assumed given (e.g. radiation from an antenna) this usually is, in fact, the equation solved to obtain $\boldsymbol{E}$. In a plasma, however, where $\boldsymbol{J}$ cannot be assumed known independently of $\boldsymbol{E}$, Eq. (6) may be used to derive an important approximation to the exact generalized Ohm's law (5).

For economy of notation, rewrite Eq. (5) as

$$
\partial \boldsymbol{J} / \partial t=\left(\omega_{p}^{2} / 4 \pi\right)\left(\boldsymbol{E}-\boldsymbol{E}^{*}\right)
$$

by lumping together all the terms other than $\boldsymbol{E}$ on the righthand side of (5) under the single symbol $\boldsymbol{E}^{*}$ defined by

$$
\begin{gathered}
-\left(\omega_{p}^{2} / 4 \pi\right) \boldsymbol{E}^{*} \equiv \sum_{a}\left\{\left(q_{a}^{2} n_{a} / m_{a}\right)\left(\boldsymbol{V}_{a} \times \boldsymbol{B} / c\right)\right. \\
\left.-\left(q_{a} / m_{a}\right) \nabla \cdot \boldsymbol{\kappa}_{a}+q_{a} n_{a} \mathbf{g}\right\}+(\delta \boldsymbol{J} / \delta t)_{c o l l}
\end{gathered}
$$

where

$\omega_{p}^{2}=4 \pi \sum_{a} q_{a}^{2} n_{a} / m_{a} \approx 4 \pi n_{e} e^{2} / m_{e}$.

is equal (except for negligibly small $m_{e} / m_{i}$ corrections) to the (electron) plasma frequency. Now replace $\partial \boldsymbol{J} / \partial t$ by the expression on the right-hand side of Eq. (6) and divide by $\omega_{p}^{2} / 4 \pi$ to rewrite the generalized Ohm's law as

$-\lambda_{e}^{2} \nabla \times(\nabla \times \boldsymbol{E})-\left(1 / \omega_{p}^{2}\right) \partial^{2} \boldsymbol{E} / \partial t^{2}=\boldsymbol{E}-\boldsymbol{E}^{*}$

where

$\lambda_{e} \equiv\left(m_{e} c^{2} / 4 \pi n_{e} e^{2}\right)^{1 / 2}=5 \mathrm{~km}\left(1 \mathrm{~cm}^{-3} / n_{e}\right)^{1 / 2}$

is the electron inertial length.

A comparison of orders of magnitude now shows that the left-hand side of Eq. (9) is non-negligible in comparison with the term $\boldsymbol{E}$ on the right-hand side only when the electric field varies on time scales as fast as or faster than $1 / \omega_{p}$ or on spatial scales as short as or shorter than $\lambda_{e}$. As far as any phenomena on time scales much slower than and spatial scales much longer than the above are concerned, the left-hand side of Eq. (9) is completely negligible. This implies, however, that the corresponding term $\partial \boldsymbol{J} / \partial t$ in Eq. (7) or (5) is likewise negligible (Vasyliūnas, 1996; with reference to time scales only, this conclusion was stated already by Dungey, 1958).

There is a subtle difficulty with the above order-ofmagnitude argument. Except for relativistic corrections of order $(v / c)^{2}$ and charge-density effects, $\partial \boldsymbol{J} / \partial t$ is independent of frame of reference and hence so is the differential 
operator on $\boldsymbol{E}$ expressed by the left-hand side of Eq. (9). The value of $\boldsymbol{E}$ itself, however, depends strongly on choice of frame of reference. Arguing that the left-hand side of Eq. (7) can be neglected because it is small compared to the first term on the right-hand side may thus seem questionable when, by choosing an appropriate frame of reference, one can make that first term small or even zero at any particular point (albeit not everywhere). A more precise and formal argument is needed. Write

$\boldsymbol{E}=\boldsymbol{E}^{*}+\delta \boldsymbol{E}$

where $\boldsymbol{E}^{*}$ is defined by Eq. (8). The generalized Ohm's law then becomes

$\partial \boldsymbol{J} / \partial t=\left(\omega_{p}^{2} / 4 \pi\right)\left(\boldsymbol{E}-\boldsymbol{E}^{*}\right)=\left(\omega_{p}^{2} / 4 \pi\right) \delta \boldsymbol{E}$

and the question of neglecting $\partial \boldsymbol{J} / \partial t$ becomes that of neglecting $\delta \boldsymbol{E}$. From Eqs. (9) and (10) it follows that the evolution of $\delta \boldsymbol{E}$ is governed by

$$
\begin{gathered}
c^{2} \nabla \times(\nabla \times \delta \boldsymbol{E})+\partial^{2} \delta \boldsymbol{E} / \partial t^{2}+\omega_{p}^{2} \delta \boldsymbol{E}= \\
-c^{2} \nabla \times\left(\nabla \times \boldsymbol{E}^{*}\right)-\partial^{2} \boldsymbol{E}^{*} / \partial t^{2}
\end{gathered}
$$

which is the plasma wave equation, with the source of the waves (right-hand side) given by the spatial and temporal second derivatives of $\boldsymbol{E}^{*}$. The presence of the plasma frequency term in Eq. (11) ensures that the typical amplitude of $\delta \boldsymbol{E}$ is related to the typical range of variation of $\boldsymbol{E}^{*}$ by the factors

$\left(\lambda_{e} / \mathcal{L}\right)^{2}, \quad\left(1 / \tau \omega_{p}\right)^{2}$

where $\mathcal{L}$ and $\tau$ are typical spatial and temporal scales of the variation of $\boldsymbol{E}^{*}$. If

$\lambda_{e} / \mathcal{L} \ll 1$ and $1 / \tau \omega_{p} \ll 1$

then $\boldsymbol{E}=\boldsymbol{E}^{*}$ to second order in small quantities, which is equivalent to neglecting the $\partial \boldsymbol{J} / \partial t$ term in the generalized Ohm's law.

I shall refer to the spatial and temporal scales defined by the inequalities (12) - in practice, all scales larger than those of electron plasma oscillations - as the large-scale or plasma limit; this is the regime in which any difference in concentration between the positive and the negative charged particles must be small in comparison to the concentration of either one, and the behavior of the plasma is thus strongly constrained by quasineutrality. In this limit the generalized Ohm's law reduces to $\boldsymbol{E}=\boldsymbol{E}^{*}$ or, written out in full,

$$
\begin{aligned}
0= & \sum_{a}\left\{\left(q_{a}^{2} n_{a} / m_{a}\right)\left(\boldsymbol{E}+\boldsymbol{V}_{a} \times \boldsymbol{B} / c\right)\right. \\
& \left.-\left(q_{a} / m_{a}\right) \nabla \cdot \boldsymbol{\kappa}_{a}+q_{a} n_{a} \mathbf{g}\right\}+(\delta \boldsymbol{J} / \delta t)_{\text {coll }} .
\end{aligned}
$$

\subsection{Equations for $\boldsymbol{E}$ and $\boldsymbol{J}$}

The preceding development has shown that the generalized Ohm's law, fundamentally the evolutionary equation giving the time derivative of $\boldsymbol{J}$, in the large-scale plasma limit becomes instead the equation that directly gives $\boldsymbol{E}$ (and therefore also its time history). This immediately raises two questions: 1) How is the $\boldsymbol{E}$ so determined to be reconciled with its own evolutionary Eq. (3)? 2) What, then, determines $\boldsymbol{J}$ ? Traditionally both questions are evaded together by assuming (e.g. Vasyliūnas, 1996) that the displacement current, being closely connected with charge separation, must become negligible under the assumption of quasineutrality and hence Eq. (3) must reduce to

$\boldsymbol{J}=(c / 4 \pi) \nabla \times \boldsymbol{B}$

This argument fails, however, whenever the nominal Alfvén speed is comparable to or larger than the speed of light; the displacement current cannot then be neglected (e.g. Boris, 1970; Gombosi et al., 2002), regardless of any quasineutrality considerations.

The problem can be solved by a method similar to that used above for the generalized Ohm's law. Write

$\boldsymbol{J}=\boldsymbol{J}^{*}+\delta \boldsymbol{J}$

where $\boldsymbol{J}^{*}$ is defined by setting

$4 \pi \boldsymbol{J}+\partial \boldsymbol{E} / \partial t=4 \pi \boldsymbol{J}^{*}+\partial \boldsymbol{E}^{*} / \partial t$

with $\boldsymbol{E}^{*}$ still defined by Eq. (8). Then

$4 \pi \delta \boldsymbol{J}+\partial \delta \boldsymbol{E} / \partial t=0$

and from Eqs. (10) and (16) one obtains the equation for the evolution of $\delta \boldsymbol{J}$

$\partial^{2} \delta \boldsymbol{J} / \partial t^{2}+\omega_{p}^{2} \delta \boldsymbol{J}=-\partial^{2} \boldsymbol{J}^{*} / \partial t^{2}$

which is again the plasma wave equation, similar to but simpler than Eq. (11), with the source given by the second time derivative of $\boldsymbol{J}^{*}$. In the limit defined by the inequalities (12), it follows (by the same argument as for $\boldsymbol{E}=\boldsymbol{E}^{*}$ ) that $\boldsymbol{J}=\boldsymbol{J}^{*}$. The equation that determines $\boldsymbol{J}$ then becomes

$\boldsymbol{J}=(c / 4 \pi) \nabla \times \boldsymbol{B}-(1 / 4 \pi) \partial \boldsymbol{E} / \partial t$

identical in form to Eq. (3) but different in interpretation: it is now the equation that directly gives $\boldsymbol{J}$, modified if necessary by the displacement current term $\partial \boldsymbol{E} / \partial t$ (which has already been fixed by the time profile of $\boldsymbol{E}$ from the generalized Ohm's law).

To summarize: on all scales larger than those of electron plasma oscillations, neither the time evolution of $\boldsymbol{J}$ nor that of $\boldsymbol{E}$ can be calculated directly, the time derivatives being either negligible in comparison to the other terms of the nominal time-evolution equation or else fixed by other equations. Instead, $\boldsymbol{E}$ is determined by plasma dynamics through the generalized Ohm's law (13) and $\boldsymbol{J}$ is determined by $\boldsymbol{B}$ through Ampère's law (14) (or Maxwell's equation (18) if the modification of $\boldsymbol{J}$ by the displacement current is significant). 


\subsection{Application to multiscale systems}

Plasmas in general and space plasmas in particular are often characterized by the simultaneous occurence of phenomena on different spatial and temporal scales, ranging from the smallest to the largest. It is therefore a valid question how an approximation valid only for large scales can be applied in a situation where large and small scales are both present.

Any quantity that is a function of space and time, including the velocity distribution functions $f_{a}$ and their moments as well as $\boldsymbol{E}$ and $\boldsymbol{B}$, can be averaged over a time scale $\gg 1 / \omega_{p}$ and a spatial scale $\gg \lambda_{e}$; let \langle\rangle$=$ average and $\delta=$ deviation from average (note that $\delta$ here is not the same as that used in Eqs. (10) to (17)). The generalized Ohm's law Eq. (5) is linear in the moments of the distribution functions and also linear in the electric and magnetic fields but contains terms with products of moment and field. Averaged, it becomes

$$
\begin{aligned}
0 & =\sum_{a}\left\{\left(q_{a}^{2}\left\langle n_{a}\right\rangle / m_{a}\right)\left(\langle\boldsymbol{E}\rangle+\left\langle\boldsymbol{V}_{a}\right\rangle \times\langle\boldsymbol{B}\rangle / c\right)\right. \\
& -\left(q_{a} / m_{a}\right) \nabla \cdot\left\langle\boldsymbol{\kappa}_{a}\right\rangle+q_{a}\left\langle n_{a}\right\rangle \mathbf{g} \\
& \left.+\left(q_{a}^{2} / m_{a}\right)\left(\left\langle\delta n_{a} \delta \boldsymbol{E}\right\rangle+\left\langle\delta\left(n_{a} \boldsymbol{V}_{a}\right) \times \delta \boldsymbol{B}\right\rangle / c\right)\right\} \\
& +\left\langle(\delta \boldsymbol{J} / \delta t)_{\text {coll }}\right\rangle .
\end{aligned}
$$

The left-hand side is actually $\partial\langle\boldsymbol{J}\rangle / \partial t$ but has been set to zero in accordance with the previous discussion, noting that Eq. (6) is linear, hence valid also when averaged, and that inequalities (12) hold automatically for the averaged quantities. Otherwise, Eq. (19) is exact; note that $\left\langle\boldsymbol{V}_{a}\right\rangle$ is not the average of bulk velocities but must be defined as

$\left\langle\boldsymbol{V}_{a}\right\rangle=\left\langle n_{a} \boldsymbol{V}_{a}\right\rangle /\left\langle n_{a}\right\rangle$

and similarly for any decomposition of the kinetic tensors into flow and pressure components.

Equation (19) is identical with the large-scale limit Eq. (13) of the generalized Ohm's law except for the additional terms of averaged products of deviations, $\langle\delta . . \delta .$.$\rangle .$ These represent the effects of any small-scale phenomena such as microinstabilities and turbulence and must be calculated from corresponding specific models. In simple cases (e.g. Yoon and Lui, 1998; Lui, 2000) they can be represented as an effective ("anomalous") resistivity proportional to (as the $\langle\delta . . \delta .$.$\rangle form suggests) the mean square amplitude of the$ fluctuating small-scale fields.

\subsection{Physical description}

The physical reason why in the large-scale plasma limit the equation that nominally should determine $\partial \boldsymbol{J} / \partial t$ serves instead to determine $\boldsymbol{E}$ lies in the constraint of plasma quasineutrality. With nearly equal concentrations of positive and negative charged particles, the current arises from a difference in their bulk velocities, and a change of current arises from a difference of forces (each multiplied by the particle charge/mass ratio) acting on them. The imbalance of forces must not, however, be so large as to result in completely different displacements of positive and negative charged particles, inconsistent with the quasineutrality constraint. The electric field is the only quantity that can adjust itself to limit the net imbalance as required, the other forces being determined already by the bulk flows and kinetic tensors of the different species. The essential physical meaning of the generalized Ohm's law is thus that $\boldsymbol{E}$ assumes the value it must have in order to prevent a differential acceleration of ions and electrons that would separate charges too much.

Strictly speaking, the constraint of plasma quasineutrality is just an aspect of (and not really distinct from) the largescale plasma limit approximation to the generalized Ohm's law; it merely happens to be much better known. If we take the divergence of Eq. (7) and invoke Maxwell's equations (or equivalently take the divergence of Eq. (9) directly), we obtain

$$
-\left(1 / \omega_{p}^{2}\right) \partial^{2} 4 \pi \rho_{c} / \partial t^{2}=4 \pi \rho_{c}-\nabla \cdot \boldsymbol{E}^{*}
$$

where $\rho_{c}$ is the charge density. The left-hand side of Eq. (20) can be neglected, by the same arguments as before, on time scales long compared to $1 / \omega_{p}$. Comparison with the electron kinetic tensor term within $\nabla \cdot \boldsymbol{E}^{*}$ on the right-hand side then leads to the well known result that $\left|\rho_{c}\right| / e n_{e} \ll 1$ on spatial scales large compared to the Debye length.

\section{The plasma momentum equation}

The current density appears also in the momentum equation for the plasma as a whole, which for our purposes can be written as

$\partial \rho \boldsymbol{V} / \partial t=-\nabla \cdot \boldsymbol{\kappa}+\boldsymbol{J} \times \boldsymbol{B} / c+(\delta \rho \boldsymbol{V} / \delta t)_{p n}$,

exact except for neglect of electric-field terms, assuming charge quasineutrality (and $V^{2} \ll c^{2}$ ). Here $\rho$ is the mass density, $\boldsymbol{V}$ is the bulk velocity, and

$\boldsymbol{\kappa}=\sum_{a} \boldsymbol{\kappa}_{a}=\rho \boldsymbol{V} \boldsymbol{V}+\mathrm{P} ;$

a collision term $(\delta \rho \boldsymbol{V} / \delta t)_{p n}$ has been included, applicable primarily in the ionosphere, representing momentum transfer by collisions between plasma and neutral particles only (collisions of charged particles among themselves do not affect the momentum of the plasma).

In many contexts, Eq. (21) is used to obtain currents and also their time variation, by neglecting the time derivative on the left-hand side and then calculating $J_{\perp}$ from stress balance. Conventional magnetosphere/ionosphere coupling theory (e.g. Vasyliūnas, 1970, 1972; Wolf, 1983) is a particularly well known example. Less well known is the fact that, as shown by Vasyliūnas and Song (2005) on the basis of earlier work by Song et al. (2001), the ionospheric Pedersen and Hall currents are also derived from the plasma momentum equation (rather than being, as often supposed, Ohmic currents in the conventional sense): they arise from stress balance of $\boldsymbol{J} \times \boldsymbol{B} / c$ against friction of differential bulk flow between ions and neutral particles. Note that such a direct calculation of the current from the momentum equation is possible only when the magnetic field is nearly curl-free and 
the current represents a small perturbation (in practice, only within the ionosphere and the inner magnetosphere). In the general case, one must first calculate the magnetic field itself, solving the equation

$\nabla \cdot \boldsymbol{\kappa}=(\nabla \times \boldsymbol{B}) \times \boldsymbol{B} / 4 \pi$

with $\boldsymbol{J}$ then obtained from $\boldsymbol{B}$ by Ampère's law Eq. (14).

Another restriction on the use of the plasma momentum Eq. (21) to determine the current is the need to neglect the time derivative on its left hand-side, thereby assuming stress balance. Fundamentally, Eq. (21) is the evolutionary equation for plasma bulk flow, showing that the bulk acceleration of the plasma is determined by the difference between the mechanical and the magnetic stresses. Only if the difference remains small in comparison with either of the two stresses taken by itself can the acceleration term in Eq. (21) be neglected; this can be assumed only if the acceleration resulting from any imbalance between mechanical and magnetic stresses produces a flow that acts to reduce the imbalance, and only over time scales long enough for the imbalance to become negligible - in other words, the system must be stable and must evolve slowly, over time scales longer than a characteristic time easily shown to be of the order of the Alfvén wave travel time across a typical spatial scale (e.g. along a field line).

\section{Some examples}

The notion that calculating $\partial \boldsymbol{J} / \partial t$ directly (as distinct from first calculating $\nabla \times \boldsymbol{B}$ and then taking its time derivative) is not always possible may seem rather counterintuitive to some, and it is useful to illustrate it by a few simple examples. It is convenient to treat a plasma containing only electrons and one species of singly charged ions with $m_{i} \gg m_{e}$, for which the (exact) generalized Ohm's law (5) can be written as

$$
\begin{aligned}
& \partial \boldsymbol{J} / \partial t=\left(\omega_{p}^{2} / 4 \pi\right)(\boldsymbol{E}+\zeta \boldsymbol{V} \times \boldsymbol{B} / c)+\Omega_{e}^{*} \times \boldsymbol{J} \\
& \quad+e\left(\nabla \cdot \boldsymbol{\kappa}_{e} / m_{e}-\nabla \cdot \boldsymbol{\kappa}_{i} / m_{i}\right)+e\left(n_{i}-n_{e}\right) \mathbf{g} \\
& \quad+(\delta \boldsymbol{J} / \delta t)_{\text {coll }}
\end{aligned}
$$

where

$\Omega_{e}^{*} \equiv \Omega_{e}-\Omega_{i} \approx \Omega_{e}=e \boldsymbol{B} / m_{e} c$

and

$\zeta \equiv \frac{n_{i} m_{i}+n_{e} m_{e}}{n_{e} m_{i}+n_{i} m_{e}} \approx \frac{n_{i}}{n_{e}} \approx 1$

\subsection{Gyrating (electron) current}

A superficial look at Eq. (23) might suggest that a typical time scale for changes of $\boldsymbol{J}$ is the electron gyrofrequency $\Omega_{e}$. More specifically, if spatial gradients are assumed negligible (local quasi-uniformity) and $c \boldsymbol{E}+\boldsymbol{V} \times \boldsymbol{B} \approx 0$, Eq. (23) seems to indicate that any existing $\boldsymbol{J}$ will gyrate at the frequency $\Omega_{e}$; this is inconsistent, if $\Omega_{e} \ll \omega_{p}$ as is often the case, with our result that the time variation of $\boldsymbol{J}$ should not be determinable from the generalized Ohm's law.

The apparent inconsistency is resolved by noting that, in addition to Eq. (23), $\boldsymbol{J}$ always must satisfy Maxwell's equation (3); a gyrating $\boldsymbol{J}$ must be accompanied by a gyrating $\partial \boldsymbol{E} / \partial t$ or a gyrating $c \nabla \times \boldsymbol{B}$ or a combination of both. Any of these alternatives, however, implies a changing electric field that deviates from the assumed $c \boldsymbol{E}+\boldsymbol{V} \times \boldsymbol{B} \approx 0$ condition (the plasma bulk flow $\boldsymbol{V}$, dominated by the more massive ions, will not change at anything like the electron gyrofrequency) and hence adds a non-zero first term to the right-hand side of Eq. (23). In the case of $\partial \boldsymbol{E} / \partial t$ the changing electric field is given directly; in the case of $c \nabla \times \boldsymbol{B}$ it arises because a gyrating (hence time-varying) $\boldsymbol{B}$ requires a gyrating $c \nabla \times \boldsymbol{E}$ by virtue of Eq. (2). The ratio of the magnitude of the added first term to $\left|\Omega_{e} \times \boldsymbol{J}\right|$ is easily shown to be of order $\left(\omega_{p} / \Omega_{e}\right)^{2}$ in the first case and $\left(\mathcal{L} / \lambda_{e}\right)^{2}$ in the second. If the inequalities (12) which define the large-scale plasma limit hold (with $\tau=1 / \Omega_{e}$ as assumed), the added term is in either case much larger than $\left|\Omega_{e} \times \boldsymbol{J}\right|$, Eq. (23) is not satisfied, and the initial assumption that it can give the time variation of $\boldsymbol{J}$ is not valid.

This example shows that both inequalities (12) must be satisfied in order for the term $\partial \boldsymbol{J} / \partial t$ in the generalized Ohm's law to be negligible. It may be possible for $\boldsymbol{J}$ to gyrate as suggested by the superficial application of Eq. (23) even when $\Omega_{e} \ll \omega_{p}$, provided there is an associated spatial variation on a scale $\mathcal{L} \ll \lambda_{e}$, with Maxwell's equation (3) then satisfied by $\boldsymbol{J}$ and $c \nabla \times \boldsymbol{B}$ alone to order $\left(\mathcal{L} / \lambda_{e}\right)^{2}$.

\subsection{Current "disruption"}

The idea of explaining observed large-scale changes of the magnetic field configuration by some suitable local condition that changes the electric current directly through impeding its flow was proposed by Alfvén and Carlqvist (1967) in connection with solar flares. For the Earth's magnetosphere, this general concept has been subsequently developed by various authors into what are collectively called current "disruption" models for the expansion phase of magnetospheric substorms (see Lui, 1996; Birn and Hesse, 2000, and references therein): fluctuating turbulent electromagnetic fields, resulting from some specified instability which develops in conjunction with substorm onset, are assumed to reduce the current. Formally such an effect is represented in the generalized Ohm's law by the averaged quadratic $\langle\delta . . \delta .$.$\rangle terms$ that appear in Eq. (19); sometimes it is approximated by an effective (or "anomalous") collision frequency $v_{c}$, adding a term $-v_{c} \boldsymbol{J}$ to the right-hand side of the generalized Ohm's law. (Only in the simplest cases, of course, is this expected to be an adequate representation; there is no general reason for the term in question to be in the direction of $\boldsymbol{J}$ or proportional to it.) The quantity $v_{c}$ must be calculated from a specific model of the fluctuations.

As an example, Lui et al. (1993) estimate, from a quasilinear treatment of a particular cross-field current instability, the time scale $1 / v_{c}$ on which the current (assumed to exceed 
initially the threshold for instability) is reduced by the action of the unstable waves alone; they then suggest that $1 / \nu_{c}$ is to be identified with the observed time scale of dipolarization of the magnetic field. This is equivalent to keeping the $\partial \boldsymbol{J} / \partial t$ term in the generalized Ohm's law and equating it to $-v_{c} \boldsymbol{J}$. The instability considered by Lui et al. (1993) presupposes a thin current sheet, but the relevant spatial scale is comparable to the ion gyroradius and hence much larger than the electron inertial length; the time scales of their fluctuations are in the vicinity of the ion gyroperiod, very long in comparison to $1 / \omega_{p}$. The conditions (12) for the plasma limit are thus amply satisfied in this case, and again the time variation of $\boldsymbol{J}$ should not be determinable from the generalized Ohm's law - in disagreement with calculations such as that by Lui et al. (1993), representative of many others.

Here again, the apparent inconsistency is resolved by invoking Maxwell's equations to show that if $\boldsymbol{J}$ does vary on a time scale $1 / v_{c}$, this implies an electric field that, contrary to initial assumption, cannot be neglected in the generalized Ohm's law because the term it contributes is larger than $\left|v_{c} \boldsymbol{J}\right|$ by factors of order $\left(\mathcal{L} / \lambda_{e}\right)^{2}$ or $\left(\omega_{p} / v_{c}\right)^{2}$. (Note that if $1 / v_{c}$ is long enough, the change of the plasma bulk flow $\boldsymbol{V}$, influenced by $\boldsymbol{J}$ through the momentum Eq. (21), may also be significant). Vasyliūnas (1996) showed that, following the onset of an effective current-reducing process, the time scale for the subsequent evolution of $\nabla \times \boldsymbol{B}$ and hence of $\boldsymbol{J}$ is not directly related to $1 / \nu_{c}$ but rather is comparable (at least in order of magnitude) to the Alfvén wave travel time, a conclusion supported by more detailed numerical modeling (see Birn and Hesse, 2000, and references therein).

\section{Implications for theories of magnetospheric sub- storms}

The evolution of the magnetic field during the expansion phase is one of the most striking and, despite decades of research, still not fully understood aspects of the magnetospheric substorm. The basic observed phenomenon is what is often called "dipolarization": the magnetic field in the middle and outer magnetosphere becomes more dipole-like over a limited local time sector, while still remaining taillike in adjacent local times. Dipolarization begins typically near the midnight meridian at a radial distance $\sim 6-7 R_{E}$ and then propagates westward and eastward as well as tailward (see Ohtani et al., 1991, and references therein). The overall change occurs on a time scale of a few minutes and may be accompanied by fluctuations on shorter time scales ranging down to and even below the ion gyrofrequency (Lui et al., 1992).

The phenomenon of dipolarization is often described as the formation and evolution of an (inferred) substorm current wedge (McPherron et al., 1973): the cross-tail current is reduced over the limited local time sector by having part of the current flow down along magnetic field lines to the ionosphere, westward across the ionosphere, and back up along the field lines. (Note the "inferred": all statements about ob- served currents are inferences from observations of the magnetic field - there are no direct observations of currents of any significance.) On the theoretical side, many of the attempts at interpretation and explanation are likewise formulated in terms of currents: the aim is to find a physical model that would predict the formation and subsequent evolution of the current wedge, from which the dipolarization of the magnetic field could then be deduced.

The results in the present paper imply that any such theoretical model of dipolarization, in terms of the current as the primary quantity, is not possible: On time scales appropriate to substorm expansion, there is no equation from which the time evolution of the current could be calculated, prior to and independently of $\nabla \times \boldsymbol{B}$. The observed time scales typical of substorm onset and expansion are much longer than the electron plasma period, and the spatial scales are much larger than the electron inertial length; thus the large-scale plasma limit applies, where $\partial \boldsymbol{J} / \partial t$ cannot be calculated from the generalized Ohm's law. But also, the time scales are shorter than or at most comparable to the Alfvén wave travel time; thus $\boldsymbol{J}$ or $\partial \boldsymbol{J} / \partial t$ cannot be calculated from the momentum (stress balance) equation, either. (Furthermore, using stress balance to determine $\boldsymbol{J}$ presupposes that the system is stable, whereas substorm onset is widely believed to result from some type of instability.)

These limitations apply to any attempts at accounting for changing magnetic fields by invoking changing currents current disruption, diversion, wedge formation, etc. Over the wide range of time scales from electron plasma period to Alfvén wave travel time, there simply is no way to calculate the changing currents except by taking the curl of the changing magnetic fields; statements about changes of current are not explanations but merely descriptions of changes in the magnetic field. The physically meaningful approach to explaining changes of the magnetic field on substorm onset time scales is to note, first, that $\partial \boldsymbol{B} / \partial t$ is determined by $\nabla \times \boldsymbol{E}$, according to Eq. (2). In turn, $\boldsymbol{E}$ can be calculated only from the generalized Ohm's law (13) or (19) and thus is controlled by plasma flow and dynamics. Any effects of microinstabilities and turbulence are represented by the averaged products of fluctuations (the $\langle\delta . . \delta .$.$\rangle terms) in Eq. (19).$

\section{Summary and conclusions}

The equations of classical physics uniquely determine the time evolution of any quantity in terms of quantities at a given time. The time evolution of $\boldsymbol{B}$ is determined by $-\nabla \times \boldsymbol{E}$ (Eq. (2), Faraday's law). The time evolution of $\boldsymbol{E}$ is determined by the difference between $\nabla \times \boldsymbol{B}$ and $(4 \pi / c) \boldsymbol{J}$ (Eq. (3), Ampère's law as modified by Maxwell), and the time evolution of $\boldsymbol{J}$ is determined by the sum of charge times acceleration of all particles, expressible in terms of appropriate moments of the particle velocity distribution functions (Eq. (5), generalized Ohm's law in its fundamental exact form). The evolutionary equations for $\boldsymbol{E}$ and for $\boldsymbol{J}$, however, are closely coupled: the first term of the equation for 
$\partial \boldsymbol{J} / \partial t)$ is $\left(n_{e} e^{2} / m_{e}\right) \boldsymbol{E}$, which, if not balanced by the remaining terms, leads to a rapid change of $\boldsymbol{J}$ (acceleration of electrons), and the resulting $\boldsymbol{J}$ in turn, if not balanced by $\nabla \times \boldsymbol{B}$ in Maxwell's equation (3), leads to a rapid change of $\boldsymbol{E}$ (displacement current). A quantitative treatment shows that these changes proceed on time scales comparable to the inverse electron plasma period and spatial scales comparable to the electron inertial length; on all larger scales, $\boldsymbol{E}$ has adjusted itself to the value at which it is effectively in balance with the other terms of the generalized Ohm's law (including any nonlinear averaged contributions of small-scale fluctuations), and the $\partial \boldsymbol{J} / \partial t$ ) term has become negligible in comparison.

As a consequence, in the large-scale plasma limit the time evolution of electric fields and currents is no longer described by evolutionary equations giving their time derivatives as functions of the present values (including spatial derivatives). Instead, the present value - not the time derivative - of the electric field is given (as function of other present values) by the generalized Ohm's law without the $\partial \boldsymbol{J} / \partial t$ term, and the present value of the current density is similarly given by Ampère's law (including if necessary the displacement current from the already-determined electric field). This does not mean that the fundamental evolutionary equations for $\boldsymbol{E}$ and $\boldsymbol{J}$ are no longer valid: they do remain valid, but the coupled changes they imply occur at the plasma oscillation scale and therefore are effectively instantaneous if viewed on larger scales.

That $\boldsymbol{E}$ and $\boldsymbol{J}$ are secondary quantities insofar as time development is concerned, being calculated not from timederivative equations but instantaneously from other quantities which do have their own evolutionary equations, has long been a familiar concept within magnetohydrodynamics (Cowling, 1957; Dungey, 1958), recently emphasized particularly by Parker $(1996,2000)$. The work reported here shows that this concept is valid well beyond the range of MHD (generally considered no longer applicable once scales as small as ion gyroperiod or ion inertial length are approached); it applies down to the scales of electron plasma oscillations, and its limits are set by the breakdown not of the frozen-flux approximation but of charge quasineutrality.

Many aspects of the magnetosphere can be theoretically described as a sequence of equilibrium or quasi-equilibrium states; in such theories the time evolution equations are of little or no importance. For describing fast dynamical processes, however, of which the onset and expansion of the magnetospheric substorm is perhaps the most significant and spectacular example, adequate understanding and proper application of the time evolution equations, particularly those for the electric field and current as well as the magnetic field, are indispensable. The main lessons from the work reported here are the following:

1. Over the pertinent time scales, there are no equations that could be used to predict the variation of the current, as distinct from predicting the variation of the magnetic field itself. Formulations in terms of currents are mere descriptions which may be suggestive but are not explanatory.

2. There is a logical distinction between the present values and the time derivatives they determine. In particular, the time evolution of $\boldsymbol{B}$ is determined by $\nabla \times \boldsymbol{E}$ (not the other way around!)

3. The primary task in theoretical modeling of dipolarization or other fast changes of the magnetic field is to understand the plasma dynamical processes that lead, via the generalized Ohm's law, to the requisite development of $\nabla \times \boldsymbol{E}$

Acknowledgements. Many of the ideas in this paper were developed in the course of (sometimes highly controversial) discussions, particularly with A. T. Y. Lui, Paul Song, Yan Song, and R. Lysak.

Topical Editor T. Pulkkinen thanks S. W. H. Cowley, G. Siscoe and another referee for their help in evaluating this paper.

\section{References}

Alfvén, H. and Carlqvist, P.: Currents in the solar atmosphere and a theory of solar flares, Solar Phys., 1, 220-228, 1967.

Birn, J. and Hesse, M.: The current disruption myth, in: Magnetospheric Current Systems, edited by: S.-I. Ohtani, R. Fujii, M. Hesse, and R. L. Lysak, 285-294, AGU Geophysical Monograph 118, Washington, D.C., 2000.

Boris, J. P.: A physically motivated solution of the Alfvén problem, Techn. Report, NRL Memorandum Report 2167, Naval Research Laboratory, Washington, D.C., 1970.

Cowling, T. G.: Magnetohydrodynamics, Section 1.3, Interscience Publishers, Inc., New York, 1957.

Dungey, J. W.: Cosmic Electrodynamics, p.10, Cambridge University Press, London, 1958.

Eddington, A. S.: The Expanding Universe (quoted from 1958 reprint, p. 57, University of Michigan Press, Ann Arbor), Cambridge University Press, London, 1933.

Gombosi, T. I., Tóth, G., De Zeeuw, D. L., Hansen, K. C., Kabin, K., and Powell, K. G.: Semirelativistic magnetohydrodynamics and physics-based convergence acceleration, J. Computational Phys., 177, 176-205, 2002.

Greene, J. M.: Moment equations and Ohm's law, Plasma Phys., 15, 29-36, 1973.

Lui, A. T. Y.: Current disruption in the Earth's magnetosphere: Observations and models, J. Geophys. Res., 101, 13 067-13088, 1996.

Lui, A. T. Y.: Electric current approach to magnetospheric physics and the distinction between current disruption and magnetic reconnection, in: Magnetospheric Current Systems, edited by: Ohtani, S.-I., Fujii, R., Hesse, M., and Lysak, R. L., 31-40, AGU Geophysical Monograph 118, Washington, D.C., 2000.

Lui, A. T. Y., Lopez, R. E., Anderson, B. J., Takahashi, K., Zanetti, L. J., McEntire, R. W., Potemra, T. A., Klumpar, D. M., Greene, E. M., and Strangeway, R.: Current disruptions in the near-Earth neutral sheet region, J. Geophys. Res., 97, 1461-1480, 1992.

Lui, A. T. Y., Yoon, P. H., and Chang, C.- L.: Quasi-linear analysis of ion Weibel instability in the Earth's neutral sheet, J. Geophys. Res., 98, 153-163, 1993.

McPherron, R. L., Russell, C. T., and Aubry, M. P.: Satellite studies of magnetospheric substorms on 15 August 1968, 
9. Phenomenological model for substorms, J. Geophys. Res., 78, 3131-3149, 1973.

Ohtani, S., Takahashi, K., Zanetti, L. J., Potemra, T. A., McEntire, R. W., and Iijima, T. : Tail current disruption in the geosynchronous region, in: Magnetospheric Substorms, edited by: Kan, J. R., Potemra, T. A., Kokubun, S., and Iijima, T., 131-137, AGU Geophysical Monograph 64, Washington, D.C., 1991.

Parker, E. N.: The alternative paradigm for magnetospheric physics, J. Geophys. Res., 101, 10 587-10 625, 1996.

Parker, E. N.: Newton, Maxwell, and magnetospheric physics, in: Magnetospheric Current Systems, edited by: Ohtani, S.-I., Fujii, R., Hesse, M., and Lysak, R. L., 1-10, AGU Geophysical Monograph 118, Washington, D.C., 2000.

Rossi, B. and Olbert, S.: Introduction to the Physics of Space, Chapters 10 and 12, McGraw-Hill, New York, 1970.

Song, P., Gombosi, T. I., and Ridley, A. J.: Three-fluid Ohm's law, J. Geophys. Res., 106, 8149-8156, 2001.

Song, P., Vasyliūnas, V. M., and Ma, L.: A three-fluid model of solar wind-magnetosphere-ionosphere-thermosphere coupling, in: Multiscale Coupling of Sun-Earth Processes, edited by: Lui, A. T. Y., Kamide, Y., and Consolini, G., 447-456, Elsevier, Amsterdam, The Netherlands, 2005.

Vasyliūnas, V. M.: Mathematical models of magnetospheric convection and its coupling to the ionosphere, in: Particles and Fields in the Magnetosphere, edited by: McCormack, B. M., 6071, D. Reidel Publishing Co., Dordrecht-Holland, 1970.
Vasyliūnas, V. M.: The interrelationship of magnetospheric processes, in: The Earth's Magnetospheric Processes, edited by: McCormack, B. M., 29-38, D. Reidel Publishing Co., Dordrecht-Holland, 1972.

Vasyliūnas, V. M.: Time scale for magnetic field changes after substorm onset: constraints from dimensional analysis, in: Physics of Space Plasmas (1995), Number 14, edited by: Chang T. and Jasperse, J. R., 553-560, MIT Center for Geo/Cosmo Plasma Physics, Cambridge, Massachusetts, 1996.

Vasyliūnas, V. M.: Electric field and plasma flow: What drives what?, Geophys. Res. Lett., 28, 2177-2180, 2001.

Vasyliūnas, V. M. and Song, P.: Meaning of ionospheric Joule heating, J. Geophys. Res., 110( A02301), doi:10.1029/2004JA010615, 2005.

Wolf, R. A.: The quasi-static (slow-flow) region of the magnetosphere, in: Solar-Terrestrial Physics, edited by: Carovillano, R. L. and Forbes, J. M., 303-368, D. Reidel Publishing Co., Dordrecht-Holland, 1983.

Yoon, P. H. and Lui, A. T. Y.: Cross-field current instability and substorm expansion onset, in: Substorms-4, edited by: Kokubun, S. and Kamide, Y., 387-390, Terra Scientific Publishing Company/Kluwer Academic Publishers, Dordrecht, The Netherlands, 1998. 